\title{
Current concepts in hip-spine relationships: making them practical for total hip arthroplasty
}

\author{
Luigi Zagra ${ }^{1}$, Francesco Benazzo ${ }^{2}$, Dante Dallari ${ }^{3}$, Francesco Falez ${ }^{4}$, Giuseppe Solarino ${ }^{5}$, \\ Rocco D'Apolito ${ }^{1}$ and Claudio Carlo Castelli ${ }^{6}$
}

\begin{abstract}
${ }^{1}$ Hip Department, IRCCS Istituto Ortopedico Galeazzi, Milan, Italy
${ }^{2}$ Chirurgia Protesica ad Indirizzo Robotico, Fondazione Poliambulanza, Brescia, Italy

${ }^{3}$ Reconstructive Orthopaedic Surgery and Innovative Techniques - Musculoskeletal Tissue Bank, IRCCS Istituto Ortopedico Rizzoli, Bologna, Italy

${ }^{4}$ Department of Orthopaedics and Traumatology, ASL Roma 1, S. Filippo Neri Hospital, Rome, Italy

${ }^{5}$ Department of Basic Medical Sciences, Neuroscience and Sense Organs, Orthopaedic \& Trauma Unit, School of Medicine, University of Bari Aldo Moro, AOU Consorziale 'Policlinico', Bari, Italy

${ }^{6}$ FROM, Research Foundation Papa Giovanni XXIII Hospital, Bergamo, Italy
\end{abstract}

Correspondence
should be addressed
to L Zagra
Email
luigi.zagra@fastwebnet.it
- Hip, spine, and pelvis move in coordination with one another during activity, forming the lumbopelvic complex (LPC).

- These movements are characterized by the spinopelvic parameters sacral slope, pelvic tilt, and pelvic incidence, which define a patient's morphotype.

- LPC kinematics may be classified by various systems, the most comprehensive of which is the Bordeaux Classification.

- Hip-spine relationships in total hip arthroplasty (THA) may influence impingement, dislocation, and edge loading.

- Historical 'safe zones' may not apply to patients with impaired spinopelvic mobility; adjustment of cup inclination and version and stem version may be necessary to achieve functional orientation and avert complications.

- Stem design, bearing surface (including dual mobility), and head size are part of the armamentarium to treat abnormal hip-spine relationships.

- Special attention should be directed to patients with adult spine deformity or fused spine because they are at increased risk of complications after THA.

\section{Keywords}

- hip-spine

- spinopelvic mobility

total hip arthroplasty

\section{Introduction and definitions}

The hip-spine relationship has attracted increasing attention from hip surgeons, and the concept of the lumbopelvic complex (LPC) (1) has garnered research interest from diverse perspectives. This article provides an overview of the hip-spine relationship and its implications for patients undergoing total hip arthroplasty (THA).

A crucial step in THA is to position the cup in such a way to obtain a stable joint without neck-cup impingement or edge loading, which would cause abnormal wear, while maintaining an adequate range of motion (ROM) of the joint. It was long thought that inclination and anteversion as described by Lewinneck et al. (2) in 1978 could predict the risk of dislocation; indeed, they were considered 'safe zones' ( $40 \pm 10^{\circ}$ inclination and $15 \pm 10^{\circ}$ anteversion) and were the final target of cup positioning. It later emerged in most cases of dislocation, however, the socket was within the "safe zone" (3), indicating that other factors were at work. Furthermore, stem anteversion may influence the dislocation rate. Amuwa and Dorr reported a target of $35^{\circ}$ for combined anteversion (cup plus stem), with a safe zone between $25^{\circ}$ and $50^{\circ}$ (4). It has also been demonstrated that trunk equilibrium influences the $3 \mathrm{D}$ orientation of the acetabulum and the functional ROM of the hips (5).

The spine, the pelvis, and the hip move in coordination with one another during activities of everyday living, and the acetabulum changes its 3D orientation from standing to sitting or bending forward at the waist (6). The same applies to the cup once impacted. These variations can be captured on X-ray images taken in the standing and the sitting position and including the L3 vertebra to the proximal aspect of the femur (7). Spinopelvic parameters such as sacral slope (SS), pelvic tilt (PT), and pelvic incidence $(\mathrm{PI})$ aid in classifying such positional changes (Figs 1 and 2). 
The SS is the angle between the line tangent to the superior endplate of S1 and the horizontal line (8) (Fig. 1 ). The PT is the angle between the line connecting the midpoint of the sacral plate of S1 to the centre of the femoral heads and the vertical line (8) (Fig. 2). The PI, first described by Lagaye et al. (9), is the angle between the line perpendicular to the sacral plate at its midpoint and a line connecting this point to the centre of the femoral heads; it is the algebraic sum of the SS and the PT (Figs 1 and 2). It expresses the anterior to posterior dimension of the pelvis and the position of the femoral heads in relation to the spine. Philippot et al. (10) defined the PI as an 'anatomical parameter' (in contrast to the other two, which are positional parameters), because it is constant in a given patient and does not change with posture or movement.

Changing from a standing to a sitting position normally entails three movements:

\section{- $\quad$ Hip flexion}

- Reduction of lumbar lordosis (LL)

- Posterior tilt of the pelvis (decreasing the SS). The latter is called the biological opening of the acetabulum (6, 11). It results from pelvic roll-back (12) and indicates that the pelvis and the acetabulum participate together in changing their position during hip movement. This accommodates the position of the femoral neck and prevents its impingement on the anterior rim of the acetabulum (5) (increased clearance of the anterior acetabular rim). The acetabular orientation (anteversion and inclination) changes from the standing to the sitting position and vice versa (Fig. 2). Since these changes are synergistic, the combined change is termed ante-inclination (11) or functional acetabular orientation (13). In abnormal spinopelvic kinematics, these mechanisms are altered, affecting

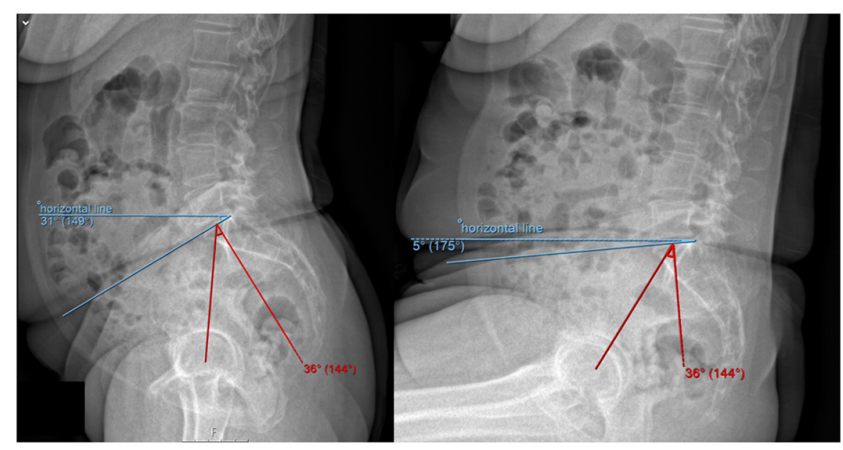

\section{Figure 1}

Reduction of sacral slope (SS, blue lines) between the standing (left) and the sitting (right) position on a lateral radiography taken with EOS. Red lines show the pelvic incidence $(\mathrm{PI})$, which remains constant.

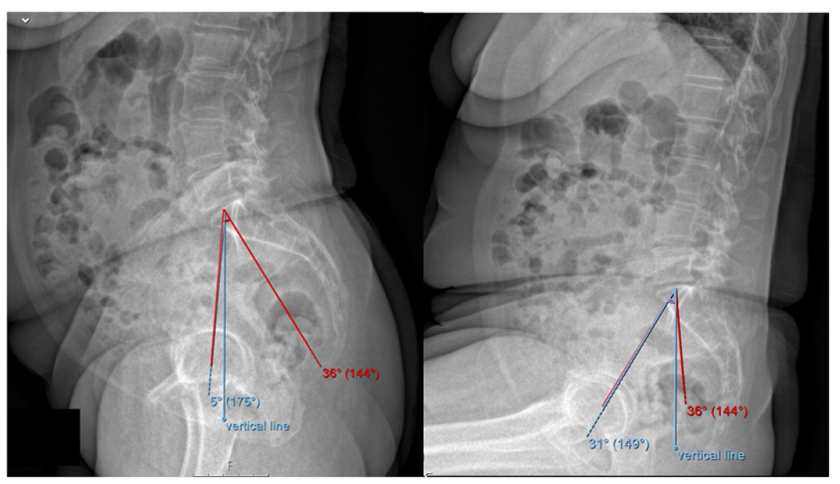

Figure 2

Increase in pelvic tilt (PT, blue lines) between the standing and the sitting position. Red lines show the pelvic incidence (PI), which remains constant.

the hip ROM and increasing the risk of impingement, dislocation, and edge loading in patients with THA.

\section{Classifications of spinopelvic deformity}

Classifying a patient's LPC kinematics is essential for the correct management of patients with concomitant hip osteoarthritis and spine pathology (14). The Bordeaux Classification of Spine-Hip Relations, devised by Rivière et al. in 2017 (1), is currently the most complete and comprehensive classification system. It describes the relationship between the hips and the spine and allows to stratify the risk of primary THA impingement or dislocation according to a patient's spine-hip relation (SHR) type (15). A lateral full spine radiograph in the standing and the sitting position is needed for classification. This can also be done with the EOS $^{\circledast}$ imaging system (EOS Imaging, Biospace ${ }^{\circledast}$, Paris, France) or with conventional radiography (16), the latter being more often available in medical centres. The aim is to evaluate PI, SS, PT, and the physiologic relationships between them $(\mathrm{PI}=\mathrm{PT}+\mathrm{SS}$ and $L L=0.54 \times \mathrm{PI}+27.6)(17)$. Classification entails the following:

1. Sagittal spine alignment according to Roussouly (18) is assessed to differentiate patients with flatback (spine types 1 and 2, with $\mathrm{Pl}<40^{\circ}$ ) from those with a more curved spine (spine types 3 and 4) (Fig. 3).

2. $\mathrm{PI}$ is evaluated.

3. Acetabular type is defined, differentiating high anteverted acetabulum (type 1) from low anteverted acetabulum (type 2 and type 3 ).

4. Evaluation of the spine sagittal profile, the PI, and the related acetabulum type differentiates LPC type 1 from LPC type 2. 


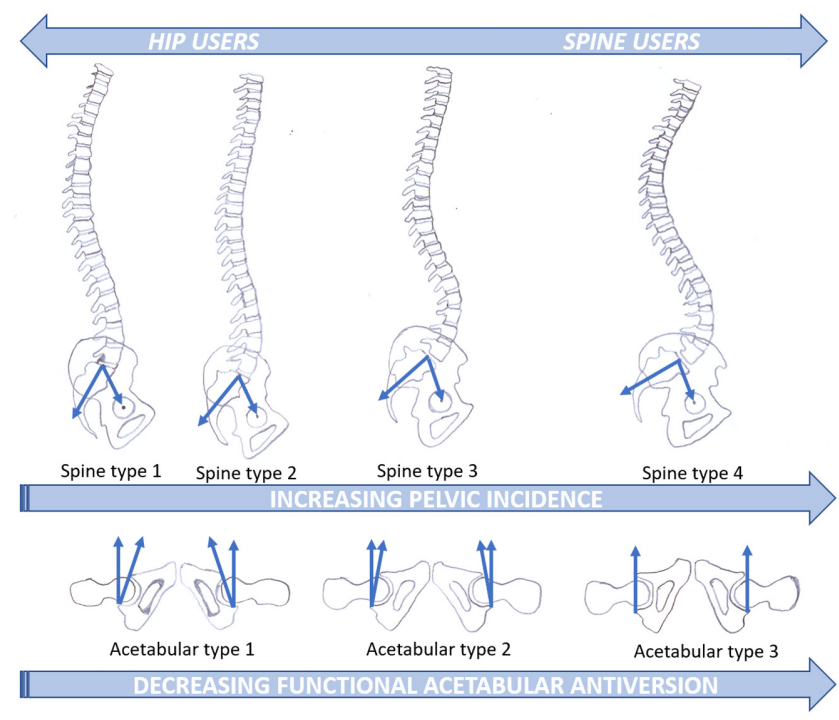

Figure 3

Four types of spine sagittal alignment according to Roussouly.

a. In LPC type 1 , the pelvis has a low $\mathrm{PI}\left(<40^{\circ}\right.$, spine type 1 or 2) and a high anteverted acetabulum (type 1). Patients with LPC type 1 tend to use a large hip cone of mobility in their daily activities (so-called 'hips users').

b. In LPC type 2 , the pelvis had a high $\mathrm{PI}\left(\geq 40^{\circ}\right.$, spine type 3 or 4) and a low anteverted acetabulum (types 2 and 3). Patients with LPC type 2 tend to have a larger pelvic ROM but a small hip cone of mobility (so-called 'spine users').

5. Modifiers A, B, C, and D are assessed (Table 1), considering the behaviour of LPC in the transition from the upright to the sitting position:

a. A: the pelvis shows $>10^{\circ}$ of retroversion when the patient is sitting.

b. B: the pelvis shows $\leq 10^{\circ}$ of retroversion when the patient is sitting.

c. C: the patient is in a compensated stage, still sagittally balanced but with a stiff LPC.

d. D: the patient is in a decompensated stage, presenting both sagittal imbalance and a very stiff LPC.

e. F: the patient has a fused spine.

Based on these findings, three risk categories are distinguished: A (very low to low risk), B and C (moderate to high risk), and $D$ and $F$ (very high risk). In summary, $A$ (in LPC type 1 or 2 ) denotes a healthy LPC, with $>10^{\circ}$ of retroversion when the patient is sitting; $B$ denotes a stiffer LPC with $<10^{\circ}$ when the patient is sitting, with increased risk of posterior edge loading, anterior impingement, and posterior dislocation when sitting or squatting; the last two categories denote spine-ageing modifications in which, in addition to a stiffer LPC, there is constant pelvic retroversion when the patient is standing while still sagittally balanced ( $C$, compensated stage) or imbalanced (D, decompensation).

The limitations of this classification system are its complexity, absence of surgical indications specific for each category (only recommendations), and the current low level of evidence (level 5, expert opinion).

In their classification system, Phan et al. (19) used the PI-LL mismatch with PT as an index of spinal sagittal balance. A multicentre study showed that the overall LL (a Cobb angle from L1 to S1) should be within $10^{\circ}$ of the PI and that the optimal spinal sagittal balance should have a PT of $<22^{\circ}$ and a PI-LL mismatch of $<11^{\circ}$ (20). Patients are classified in four categories: flexible and balanced, rigid and balanced, flexible and unbalanced, rigid and unbalanced (Fig. 4).

In a later study (7), Stefl et al. defined stuck standing (fixed anterior tilt) patients with an abnormal pattern presenting with the same characteristics as those in the rigid and balanced group and defined stuck sitting (fixed posterior tilt) patients as those similar to the rigid and unbalanced group.

More recently, Luthringer \& Vigdorchik (12) devised the Hip-Spine Classification in THA, a simplified system that identifies spinal deformity (yes or no) and spinal stiffness (yes or no), taking into account the PI-LL mismatch as a parameter of spinal deformity (Fig. 4). Patients are categorized as having:

1. Normal spinal alignment $\left(\mathrm{PI}-\mathrm{LL} \pm 10^{\circ}\right)$.

2. Flatback deformity $\left(\mathrm{PI}-\mathrm{LL}>10^{\circ}\right)$.

A. Normal spinal mobility $\left(>10^{\circ}\right.$ change in SS between the standing and the sitting position).

B. Stiff spine $\left(<10^{\circ}\right.$ change in SS between the standing and the sitting position).

Taking together the categories referring to spinal deformity ( 1 and 2 ) and spinal stiffness ( $A$ and $B$ ), four categories can be defined: 1A, 1B, 2A, and 2B (Fig. 4).

Table 1 Risk categories in the Bordeaux Classification according to LPC mobility from the standing to the sitting position.

\begin{tabular}{|c|c|c|c|c|c|}
\hline & \multicolumn{5}{|c|}{ Category } \\
\hline & $\mathrm{A}$ & B & $\mathrm{C}$ & D & $\mathbf{F}$ \\
\hline Imaging findings & $\Delta S S$ standing-sitting $>10^{\circ}$ & $\Delta S S$ standing-sitting $\leq 10^{\circ}$ & $\begin{array}{l}\text { Low standing SS, sagittally } \\
\text { balanced }\end{array}$ & $\begin{array}{l}\text { Low standing SS, sagittally } \\
\text { imbalanced }\end{array}$ & Fused spine \\
\hline Explanation & Physiologic mobility & Stiffness & Spine-ageing modifications & Spine-ageing modifications & Spine surgery \\
\hline Risk assessment & Very low-low & Moderate-high & Moderate-high & Very high & Very high \\
\hline
\end{tabular}




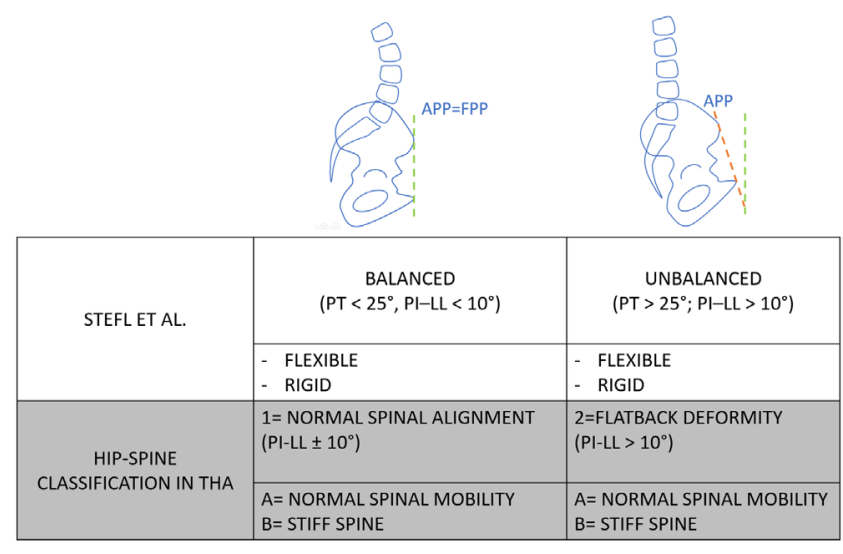

Figure 4

Classification system of Phan et al. (white boxes) and Hip-Spine Classification in THA (grey boxes). The four categories in the Phan classification are flexible and balanced (flexible spinopelvic junction and pelvic tilt $<25^{\circ}$, pelvic incidence-lumbar lordosis $<10^{\circ}$ ); rigid and balanced (rigid spinopelvic junction and pelvic tilt $<25^{\circ}$, pelvic incidence-lumbar lordosis $<10^{\circ}$ ); flexible and unbalanced (flexible spinopelvic junction and pelvic tilt $>25^{\circ}$; pelvic incidence-lumber lordosis $>10^{\circ}$ ); rigid and unbalanced (rigid spinopelvic junction and $\mathrm{PT}>25^{\circ}$; $\mathrm{PI}-\mathrm{LL}>10^{\circ}$ ). The four categories in Hip-Spine Classification are: 1A (normal alignment; normal mobility): in this type of patients, the APP and the FPP overlap in the upright position; 1B (normal alignment; stiff spine): in this type of patients, the APP and the FPP overlap in the upright position. Nonetheless, these patients have a higher risk of impingement of the flexing proximal femur on the anterior acetabular rim in the sitting position; 2A (flatback deformity; normal mobility): in these patients, the FPP is different from the APP. These patients stand with a posterior pelvic tilt because of flatback deformity, thus increasing the functional cup anteversion relative to the coronal plane while standing (FPP); $2 \mathrm{~B}$ (flatback deformity; stiff spine): in these patients, the FPP is different from the APP, and they have a stiff spine.

This classification system highlights the concepts of the anterior pelvic plane (APP, the plane between the two anterior superior iliac spines and the pubic symphysis on a lateral pelvic radiograph) and the functional pelvic plane (FPP, which is the coronal plane of the body) as references for intraoperative acetabular cup positioning (Fig. 5). The former is an anatomical parameter. In patients with no spinal deformity, it tends to be neutral and aligned with the coronal plane of the body (FPP), making it useful as a reference for cup orientation in THA. It is reported that for each degree of increased PT, there will be a concomitant increase in functional acetabular anteversion of approximately $0.7^{\circ}$ to $0.8^{\circ}$ (21). Hence, in spinal deformities, the APP reference plane could be functionally defined in a different position (i.e. tilted compared to the coronal plane) and so is an unreliable reference for acetabular component positioning. In addition, no relationships have been found between the APP and the SS in the standing position (22), whereas the SS is the pelvic functional parameter that correlates strongly with patient-
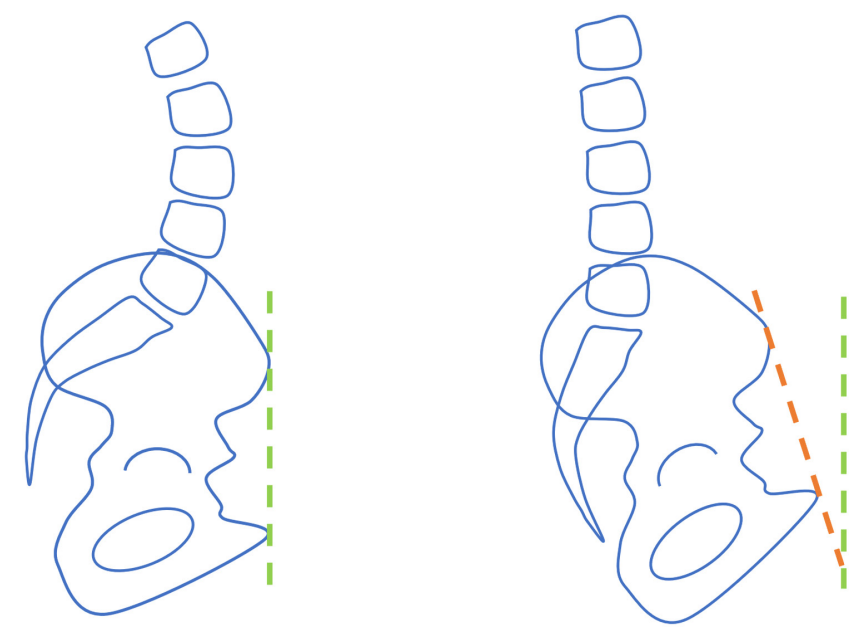

Figure 5

Anterior pelvic plane coincides with the functional pelvic plane (green line) in a healthy lumbopelvic complex and anterior pelvic plane (orange line) in a flatback deformity.

specific pelvic morphology, represented by the $\mathrm{PI}$ in the standing and the sitting position (23). Therefore, the SS has been suggested as the reference for cup adjustment during primary THA.

These classification systems provide useful information when discussing treatment with a patient with concomitant hip and spine disorders. However, future study is needed to develop a unique validated classification system, approved by both arthroplasty and spine surgeons and prospectively validated in a sufficient number of patients in order to obtain a universal surgical algorithm.

\section{Preoperative evaluation}

Medical history taking and clinical examination are the fundamental steps to identify hip and spine conditions associated with coxarthritis. Thorough history taking for temporal onset, duration, severity, and location of pain is essential. Physical examination should include observation of gait and posture when the patient is standing, flexion deformity of the hip, limb-length discrepancy, pelvic alignment (on the coronal plane for the obliquity, on the sagittal plane for the tilt), flexion, and extension of the lumbar spine (24). Sagittal imbalance, compensatory postures, scoliosis, flattening of the lumbar spine, and pelvic retroversion may be evident at clinical examination. These circumstances warrant further radiological studies in addition to the conventional workup for THA.

Imaging studies should begin with a scan in the anteroposterior (AP) and the lateral view of the pelvis in the upright position $(19,25)$, as it best reveals the patient's functional position in everyday life, even in those patients for whom 
concurrent spinopelvic conditions have been excluded. These images disclose information about the rotation and tilt of the pelvis from observation of iliac spines. They may display arthritic degeneration in the lumbar region, a possible cause of spine stiffness. A patient suffering from coxarthritis with a clinical history of spinal disorders (instability, spondylolisthesis, spondylolysis, and previous spinal surgery) (26) or positive on clinical examination for hyper/hypomobility of the spine (iatrogenic or pathologic origin) needs accurate diagnostic framing (27). In patients with a spine condition, further examination is essential for thorough pre-surgical planning to guide the surgeon in adjusting the prosthetic implant.

Imaging assessment includes scans of the pelvis and the spine in the upright position (AP and lateral views), dynamic X-ray, and EOS or CT. Based on the study findings, the parameters PT, PI, SS, and FPP can be calculated and the patients are categorized according to the abovementioned systems.

With regard to dynamic $\mathrm{X}$-rays, an overall lateral view in full-weight bearing of the lumbar spine, the pelvis, and the proximal femurs should be taken into account to determine whether degeneration or structural anomalies are present, along with a lateral view of these regions in a sitting position to evaluate dynamic changes. Lateral views in maximun flexion and extension of the lumbar spine can be useful to better evaluate lumbar stiffness or instability. Standard 36-inch cassettes are sufficient to produce useful images for evaluating the relationship between the axial and the appendicular skeleton. There are some limitations, however (11). First, the acquisitions must conform to the symmetry of the anatomical reference points in order to avoid overlapping of anatomic landmarks. Phenomena of pelvic twisting often overlap and impair evaluation of the images (28). Second, examination of 2D images could be difficult for understanding the functional evaluation of the safe zone (28), which can be better analysed by a 3D exploration.

EOS is a recent imaging technique based on the work of Georges Charpak, 1992 Nobel Prize winner for physics. $(29,30)$ It provides a static and/or functional 3D image of the skeleton. It reduces the exposure to X-ray doses compared to conventional $\mathrm{X}$-rays since it combines two simultaneous frontal and lateral acquisitions in a single scan and two gaseous detectors. EOS $\mathrm{X}$-rays and 3D reconstruction generate a full body image of the patient in the standing and the sitting position, which allows a new approach to evaluating postural abnormalities involving the spine and the subpelvic area (31).

Computed tomography (CT) scans can be used to assess the anatomical anteversion directly from crosssectional slices. However, the orientation of these slices in relation to the sagittal plane and the pelvic position during imaging acquisition are not standardized, causing a critical effect on measurement of the angles (32). Generally speaking, CT scans in the upright position overestimate acetabular anteversion, while tending to underestimate it when the patient is in a sitting position. When classic measurement of acetabular anteversion occurs, CT scans may be inadequate to determine the actual position of the acetabular cup from a functional standpoint because they are taken when the patient is in supine rather than during a dynamic change of position (33). Nonetheless, measurement of the functional anteversion corresponding to the standing and the sitting position can be done using $C T$, which requires adjustment of the sectional plane following the value of the SS on lateral radiographs with the patient in the standing and the sitting position (34). Costs and the need to assemble other scanning projections to complete the diagnostic process make CT suboptimal for analysing the characteristics of abnormal spinopelvic relations in routine practice. To overcome these limitations, specialized software is under development to create patient-specific 3D models to simulate implant fit, ROM, and impingement (35). The relevance of these 3D templating methods in reducing the risk of hip instability and dislocation, edge loading, and wear needs further clinical investigation.

\section{Implications for implant position}

Biomechanical parameters and classifications are heterogeneous; nonetheless, we can find some common 'fixed-points' on which to summarize one or more operative strategies (Table 2). Absence of spinal deformities with normal spinopelvic mobility (SS change between $10^{\circ}$ and $30^{\circ}$ from the standing to the sitting position) (7) can be safely treated with the usual indications for component positioning: cup inclination $40^{\circ}$, cup anteversion $20^{\circ}$, and femoral-cup combined anteversion $25-45^{\circ}$ (2). In these patients, the biomechanical behaviour of the LPC does not require special adjustment.

Kyphotic pelvis (PI-LL mismatch $>10^{\circ}$ ) associated with a normal spine-hip mobility usually does not require

Table 2 Cup orientation in relation to spinopelvic mobility.

\begin{tabular}{|c|c|c|c|c|}
\hline \multirow[b]{2}{*}{ Spinopelvic mobility } & \multicolumn{2}{|c|}{ Stiff } & \multirow[b]{2}{*}{ Normal } & \multirow[b]{2}{*}{ Hyper-mobile } \\
\hline & Kyphotic, pelvic incidence $>40^{\circ}$ & Pelvic incidence $<40^{\circ}$ & & \\
\hline Cup anteversion & Reduce $\left(15-20^{\circ}\right)$ & Increase $\left(20-25^{\circ}\right)$ & Usual $\left(20^{\circ}\right)$ & Reduce $\left(15-20^{\circ}\right)$ \\
\hline Cup inclination & Usual or increase & Increase $\left(45^{\circ}\right)$ & Usual $\left(40^{\circ}\right)$ & Reduce $\left(35-40^{\circ}\right)$ \\
\hline
\end{tabular}


changes in cup inclination and anteversion because of its balanced biomechanics. Hypermobility of the spinopelvic complex (SS $>30^{\circ}$ between the standing and the sitting position), particularly in a kyphotic pattern, usually requires reducing cup inclination $\left(35-40^{\circ}\right)$ and reducing cup anteversion $\left(15-20^{\circ}\right)$ to reduce the functional anteinclination in the sitting position (7).

LPC stiffness ( $\mathrm{SS}<10^{\circ}$ between the standing and the sitting position) (12) usually requires an increased cup inclination $\left(45^{\circ}\right)$ and more cup anteversion $\left(20-25^{\circ}\right)$ with a femoral-cup combined anteversion $35-50^{\circ}$ to balance the loss of posterior PT in the sitting position (7). A limited or absent pelvic roll-back in the sitting position implicates two unfavourable events: increased femoral flexion needed for a certain hip cone of mobility and reduced cup posterior protection, both leading to a potential risk of anterior impingement and posterior dislocation (12). However, SHR D patients with a severely degenerated and rigid LPC are conversely eligible for less anteverted cup positioning, especially if the $\mathrm{PI}$ is $>40^{\circ}$, as observed in LPC type 2 . The severe standing pelvic retroversion with a subsequently increased cup ante-inclination is considered at high risk for posterior impingement and anterior dislocation.

Two items to be considered in the adjustment of cup positioning are the reference point for assessing cup anteversion and how to safely change this parameter without the cup protruding from the acetabular bone profile. If a change in cup positioning has been planned, effective intraoperative control is essential to target functional cup positioning. Both the APP and the FPP are reference planes for determining cup anteversion. However, although they may be evident on a lateral projection of the pelvis, the pelvic plane may change when the patient is in supine or lateral decubitus on the operating table.

As mentioned, the APP is not a reliable reference in spinal deformities; instead, the SS is to be preferred for cup adjustment because it correlates best with the functional tilt of the pelvis (23). And while it may not be advisable to reproduce a target angle without assistive technology, the anatomical patient-specific manual technique (using the transverse acetabular ligament) may assist the surgeon in reproducing an individual acetabular anteversion that is both anatomical and functional. This is likely to be precise and reproducible in restoring patient-specific acetabular anteversion, achieving better standing cup orientation, even in dysplasia $(36,37,38,39)$. It conforms to constitutional anatomy and takes into account individual pelvic kinematics in the attempt to achieve a sort of 'kinematic alignment' (1). Moreover, it is not influenced by the patient's position on the operating table because the patient's anatomy is the only reference regardless of the sagittal and the pelvic plane. For these reasons, there may be a rationale for using this anatomical reference to guide cup version adjustment in impaired spinopelvic mobility (taking into account that it can be the expression of abnormal acetabular version in some cases). It may not always be evident, however, particularly in severe hypertrophic osteoarthritis when it is calcific.

Computer navigation for acetabular component may offer a reliable tool to carry out what was pre-operatively assessed according to spinopelvic biomechanics (7), with scrupulous attention to the reference plane since computer navigation usually relies on anatomic landmarks. Patientspecific instrumentation, with an acetabular guide created on a 3D anatomical model (after adjustment for spinopelvic mobility), may be a reliable and time-effective method to see through with the pre-operative plan. Nonetheless, correction of cup anteversion may cause the cup profile to protrude out of the anterior or posterior bone rim, leading to psoas irritation or posterior cup-neck impingement, respectively.

One way to reduce protrusion is to ream the cup further medially, while averting simultaneous superior displacement of the centre of rotation (7), which carries the risk of damaging the medial wall and reducing the global offset. Correct cup sizing can mitigate the risk of symptomatic overhang when reducing the anteversion, whereas the risk of posterior impingement can be minimized by using a bigger head. Sub-hemipherical cups may reduce protrusion of the component and increase joint ROM, but the downside is the decreased jump distance. Elevated liners have been associated with a significantly lower risk for revision THA secondary to recurrent dislocation compared to neutral liners (40), and they may help to reduce the risk of dislocation in impaired hip-spine mobility. In such instances, care is warranted to ensure that there is no component impingement that could result in liner deformation, liner fracture, or dislocation secondary to impingement in the contralateral direction. Navigation and robotics can enhance precise cup positioning, as well as successful biomechanical restoration of leg length and offset. Potential drawbacks of these technologies are the steep learning curve, plus the additional time and financial expense. Despite promising initial reports, few comparative studies have been published to date and it is unclear whether these surrogate measures will reduce revision rates or achieve better outcomes (41).

Despite our efforts to tailor cup orientation to an invidual patient, we may fail to obtain the desired cup ante-inclination or it may be insufficient to avert the risk of impingement/dislocation. In their study, Stefl et al. (7) reported abnormal postoperative functional cup positioning in $27 / 160$ hips (16.9\%), though 12 were considered inconsequential. A final point is that spine surgery may influence THA biomechanics just as hip surgery may change spinopelvic mobility. Stefl et al. (7) reported improvement in spinal mobility after release of 
hip contractures with THA and potential conversion from abnormal to normal spinal mobility also in patients with degenerative disc diseases.

\section{Implications for implant selection}

Abnormal hip-spine relationships have implications not only for implant orientation but for implant selection as well.

\section{Stem sizing, design, and fixation}

Femoral component shape, size, and position should be considered together to optimize the choice of implant (42). Preoperative planning is helpful for this purpose, in which restoration of personalized hip biomechanics is mandatory. Prevention of impingement begins with accurate reconstruction of hip length and offset. When needed, variation of the caput collum diaphyseal angle and increased offset can aid in reducing potential laxity and prevent microseparation. In patients who are stuck standing or stuck sitting, the recommendation is to increase the offset by $5 \mathrm{~mm}$ to protect against bony impingement (43). Since the orientation of the femoral component strongly affects the combined version, femoral morphotype plays a critical role, especially when uncemented stems are used. Native femoral anteversion may range from -15 (retroversion) to $30^{\circ}$ (anteversion) (44); however, fixation of an uncemented stem requires a stable press fit into the bone so that the implant can adapt to the variable femoral geometry. As a consequence, there is often less margin to adjust stem anteversion in cementless designs, particularly in femur type A and type B in the Dorr classification, regardless of their geometry (45). In detail, tapered stems have freedom of rotation of up to $5^{\circ}$, whereas metaphysealfilling stems are inflexible. High-volume experience with specific commercial cementless designs can aid in reducing unexpected version outliers (46). Differently, cemented stems can be rotated within the femoral bone to achieve 10-20 anteversion (43) and an increased combined version can be easily obtained also using cemented stems without overly resorting to version of the cup.

\section{Bearing surfaces and femoral head size selection}

THA bearing surfaces are susceptible to edge loading, and an abnormal hip-spine relationship increases its occurrence significantly. Posterior edge loading can occur during hip flexion, whereas anterosuperior edge loading can occur during full extension. A finite element study of the effect of the seated pelvis in THA demonstrated that posterior edge loading is common at relatively low angles of seated PT (47). Patient-specific cup orientation should be adjusted to minimize edge loading because it raises the contact pressure that damages the bearing surface, increasing the risk of wear and material fatigue failure. In the mid-term, this is a potential cause of adverse soft tissue reactions, osteolysis, and implant loosening. In abnormal LPC kinematics, one may ask whether edge loading of a highly cross-linked polyethylene liner is any more preferable than edge loading of a ceramic bearing. Ceramics is certainly less forgiving in suboptimal joint kinetimatics across the articular surface and excessive acetabular functional anteversion $\left(>25^{\circ}\right)$ in the standing position has been reported to be the main clinical risk factor for liner fracture due to impingement (48). In addition, edge loading is a cause of noise (squeaking) and other types of noise produced by minor joint laxity conditions like microseparation (49). Owing to the risks linked to malposition and soft tissue balancing, the use of ceramic-on-ceramic is generally contraindicated in high-risk patients (50).

Increasing the head size can improve ROM and reduce the risk of impingement and dislocation. Bigger heads lower the risk of dislocation by virtue of the increased jumping distance and better head-neck ratio (13), which remains essential and is the reason why skirted heads should be avoided. The head diameter depends on cup size and liner thickness. For polyethylene liners, a larger head size of $36 \mathrm{~mm}$ is recommended to have a minimum of thickness for fatigue resistance (50). Caution is warranted with elevated rims in impingement at-risk situations (40). Finally, too large metal head can carry the risk of corrosion at the head-neck junction, which can be eliminated using ceramic heads instead.

\section{Dual mobility}

Dual mobility constructs have been shown to reduce the risk of dislocation (51), allowing for greater joint ROM before impingement, more anatomical head size, and better head-neck ratio. The original monoblock designs have given way to modular designs in which a cobaltchromium liner is inserted into a titanium shell to obtain the familiarity of a standard titanium cup, additional fixation with screws, and the advantages of modularity. Concern has been raised, however, about the potential for fretting and corrosion between the cobalt-chromium liner and the titanium shell (52).

Dual mobility cups are indicated in patients with a stiff LPC (stuck sitting or stuck standing), plus increased femoral mobility and low PI (43). They may also be used in older patients ( $\geq 75$ years) with spinopelvic stiffness, since stiffness is likely to worsen with advancing age, increasing the need for femoral flexion, along with the greater risk of impingement (43). A systematic review of comparative studies reported evidence for the efficacy of dual mobility to minimize dislocation after THA, in addition to excellent mid-term survivorship compared to control constructs (53). Dual mobility is suggested in high-risk patients, including those with fused spine. While dual mobility 
constructs help mitigate the risk of dislocation and their use together with bigger heads has been widely accepted, accurate acetabular component positioning and refined femoral component positioning remain essential.

\section{Analysis of implant failure}

Functional acetabular orientation is useful for understanding the pathophysiology of prosthetic dislocation and is likely to explain why some patients with standard cup orientation dislocate, while others with abnormal cup orientation do not (54). Functional cup orientation is related to pelvic kinematics, which is largely influenced by lumbar mobility (55). The dislocation rate after THA is higher in patients with adult spinal deformity than in healthy controls ( $8 \%$ vs $1.5 \%$ ) (56). Spinal stiffness, whether secondary to instrumented or biologic fusion (spondylosis), imparts an equally high risk of instability after THA. Furthermore, the dislocation rate rises with the number of vertebral levels fused: $2.73 \%$ in patients with 1-2 level, $4.62 \%$ in those with 3-7 level lumbar spinal, and $1.55 \%$ in patients without spinal fusion (57). When dislocation occurs, a complete clinical and radiological workup is recommended, including $\mathrm{CT}$ and dynamic X-ray according to the protocols decribed in the paragraph on preoperative evaluation.

Failure and possible complications after spinal and hip surgery are related not only to the risk of dislocation. In their study involving 811601 US Medicare patients undergoing primary THA grouped by length of prior spinal arthrodesis (SA) (no fusion, 1-2 levels fused (S-SAHA), $\geq 3$ levels fused (L-SAHA)) and compared to controls, sing et al. found significantly higher rates of complications within 24 months in patients with prior SA $(P<0.001)$, including dislocation (controls $2.36 \%$, S-SAHA group $4.26 \%$, and L-SAHA group 7.51\%), revision (controls 3.43\%, S-SAHA group 5.55\%, and L-SAHA group 7.77\%), and implant loosening (controls $1.33 \%$, S-SAHA group $2.10 \%$, and L-SAHA group $3.04 \%$ ) (58). A retrospective study of the New York State's Department of Health database (Statewide Planning and Research Cooperative System, a payer data reporting system) showed that patients who had a long spinal fusion (four levels or more) after THA had a $340 \%$ higher odds of experiencing hip dislocation and a $220 \%$ higher odds of needing a revision THA (59). In another registry-based population study, approximately 679 (1\%) THA patients received lumbar spine fusion (LSF) and patients undergoing THA and LSF had an increased risk of mechanical complications with their THA and a slightly increased risk of revision arthroplasty (60). In their study using US Medicare data from 2005 to 2015 (42 300 patients), Malkani et al. compared the dislocation and revision risks in patients undergoing primary THA after LSF vs those undergoing THA before LSF; they reported that patients with prior LSF undergoing THA were at a far higher risk of dislocation and subsequent revision compared to those with THA followed by delayed LSF (61). The increased risk of dislocation in patients with prior LSF compared to those who had THA followed by LSF was 46,60 , and $106 \%$ within 1, 2, and 5 years, respectively. In addition, compared to those with LSF after THA, the increased revision risk for the patient group who had LSF before THA was 43,41 , and $49 \%$ within 1, 2, and 5 years, respectively. The pathogenetic mechanisms underlying the increased number of revisions are unknown. It is likely that higher risk of dislocation is linked to edge loading, with a greater risk of mechanical stress on the bone-cup interface and earlier wear of the materials.

Postoperative levels of physical activity, as measured in minutes per week, were lower in patients with prior lumbar fusion than in those without spine fusion both before and after THA surgery (62). The degree of improvement in physical activity following THA was the same for the two groups. These findings may aid in counselling patients with lumbar spine fusion and in setting appropriate expectations before they undergo THA.

Several other factors that can potentially confound study findings are the surgical approach, the type of implant, and the type of corrective spinal procedure. Further studies are desirable to determine whether surgical approach and real-time assessment of cup position using current technologies play a role in minimizing instability in this complex patient segment undergoing primary THA.

\section{Conclusion}

Hip-spine relations and their implications for planning and performing THA are attracting greater interest among orthopaedic surgeons. This update provides indications on how to interpret clinical and radiological findings and reduce the risk of complications, including implant dislocation, impingement, and wear. This patient segment is growing as the population ages. There exist several classification systems to categorize patients by specific risk based on spine-hip alignment and mobility and to adjust implant component positioning accordingly. Clinical examination and accurate evaluation of X-ray findings are crucial in patient workup. Bigger heads, dual mobility cups, and offset stems, as well as new imaging technologies, provide further tools to improve preoperative surgical planning and to achieve the desired cup and stem orientation intraoperatively.

\section{ICMJE Conflict of Interest Statement}

$\mathrm{L} Z$ has received grants for research activity not related to this article from LimaCorporate, Depuy JJ, Medacta and has received honoraria for lectures from Zimmer Biomet, LimaCorporate and Stryker; F F has received royalites not related to this article from Microport and Adler Ortho. The other authors have nothing to disclose. 


\section{Funding Statement}

This work reported here did not receive any specific grant from any funding agency in the public, commercial, or not-for-profit sector.

\section{References}

1. Rivière C, Lazennec JY, Van Der Straeten C, Auvinet E, Cobb J \& MuirheadAllwood S. The influence of spine-hip relations on total hip replacement: a systematic review. Orthopaedics and Traumatology, Surgery and Research 2017 103 559-568. (https://doi.org/10.1016/j.otsr.2017.02.014)

2. Lewinnek GE, Lewis JL, Tarr R, Compere CL \& Zimmerman JR. Dislocations after total hip-replacement arthroplasties. Journal of Bone and Joint Surgery: American Volume 197860 217-220. (https://doi.org/10.2106/00004623-197860020-00014)

3. Abdel MP, von Roth P, Jennings MT, Hanssen AD \& Pagnano MW. What safe zone? The vast majority of dislocated THAs are within the Lewinnek safe zone for acetabular component position. Clinical Orthopaedics and Related Research 2016474 386-391. (https://doi.org/10.1007/s11999-015-4432-5)

4. Amuwa C \& Dorr LD. The combined anteversion technique for acetabular component anteversion. Journal of Arthroplasty 200823 1068-1070. (https://doi.org/10.1016/j. arth.2008.04.025)

5. Lazennec JY, Riwan A, Gravez F, Rousseau MA, Mora N, Gorin M, Lasne A, Catonne Y \& Saillant G. Hip spine relationships: application to total hip arthroplasty. Hip International $2007 \mathbf{1 7}$ (Supplement 5) S91-S104.

6. Ike H, Dorr LD, Trasolini N, Stefl M, McKnight B \& Heckmann N. Spinepelvis-hip relationship in the functioning of a total hip replacement. Journal of Bone and Joint Surgery: American Volume 2018100 1606-1615. (https://doi.org/10.2106/ JBJS.17.00403)

7. Stefl M, Lundergan W, Heckmann N, McKnight B, Ike H, Murgai R \& Dorr LD. Spinopelvic mobility and acetabular component position for total hip arthroplasty. Bone and Joint Journal 2017 99-B 37-45. doi:10.1302/0301-620X.99B1.BJJ-2016-0415.R1.

8. Le Huec JC, Aunoble S, Philippe L \& Nicolas P. Pelvic parameters: origin and significance. European Spine Journal 201120 (Supplement 5) 564-571. (https://doi. org/10.1007/s00586-011-1940-1)

9. Legaye J, Duval-Beaupère $\mathbf{G}$, Hecquet $\mathbf{J}$ \& Marty C. Pelvic incidence: a fundamental pelvic parameter for three-dimensional regulation of spinal sagittal curves. European Spine Journal 1998 99-103. (https://doi.org/10.1007/s005860050038)

10. Philippot R, Wegrzyn J, Farizon F \& Fessy MH. Pelvic balance in sagittal and Lewinnek reference planes in the standing, supine and sitting positions. Orthopaedics and Traumatology, Surgery and Research 200995 70-76. (https://doi.org/10.1016/j. otsr.2008.01.001)

11. Kanawade V, Dorr LD \& Wan Z. Predictability of acetabular component angular change with postural shift from standing to sitting position. Journal of Bone and Joint Surgery: American Volume 201496 978-986. (https://doi.org/10.2106/JBJS.M.00765)

12. Luthringer TA \& Vigdorchik JM. A preoperative workup of a 'hip-spine' total hip arthroplasty patient: a simplified approach to a complex problem. Journal of Arthroplasty 201934 S57-S70. (https://doi.org/10.1016/j.arth.2019.01.012)

13. Sariali E, Lazennec JY, Khiami F \& Catonné Y. Mathematical evaluation of jumping distance in total hip arthroplasty: influence of abduction angle, femoral head offset, and head diameter. Acta Orthopaedica 200980 277-282. (https://doi. org/10.3109/17453670902988378)
14. Piazzolla A, Solarino G, Bizzoca D, Montemurro V, Berjano P, Lamartina C, Martini C \& Moretti B. Spinopelvic parameter changes and low back pain improvement due to femoral neck anteversion in patients with severe unilateral primary hip osteoarthritis undergoing total hip replacement. European Spine Journal 201827 125-134. (https://doi. org/10.1007/s00586-017-5033-7)

15. Rivière C, Lazic S, Dagneaux L, Van Der Straeten C, Cobb J \& MuirheadAllwood S. Spine-hip relations in patients with hip osteoarthritis. EFORT Open Reviews 20183 39-44. (https://doi.org/10.1302/2058-5241.3.170020)

16. Lazennec JY, Brusson A \& Rousseau MA. Hip-spine relations and sagittal balance clinical consequences. European Spine Journal 201120 (Supplement 5) 686-698. (https:// doi.org/10.1007/s00586-011-1937-9)

17. Le Huec JC \& Hasegawa K. Normative values for the spine shape parameters using 3D standing analysis from a database of 268 asymptomatic Caucasian and Japanese subjects. European Spine Journal 201625 3630-3637. doi:10.1007/s00586-016-4485-5.

18. Roussouly P \& Pinheiro-Franco JL. Biomechanical analysis of the spino-pelvic organization and adaptation in pathology. European Spine Journal $20112 \mathbf{2 0}$ (Supplement 5) 609-618. (https://doi.org/10.1007/s00586-011-1928-x)

19. Phan D, Bederman SS \& Schwarzkopf R. The influence of sagittal spinal deformity on anteversion of the acetabular component in total hip arthroplasty. Bone and Joint Journal 2015 97-B 1017-1023. (https://doi.org/10.1302/0301-620X.97B8.35700)

20. Schwab FJ, Blondel B, Bess S, Hostin R, Shaffrey Cl, Smith JS, Boachie-Adjei 0, Burton DC, Akbarnia BA, Mundis GM, et al. Radiographical spinopelvic parameters and disability in the setting of adult spinal deformity: a prospective multicenter analysis. Spine $2013 \mathbf{3 8}$ E803-E812. (https://doi.org/10.1097/ BRS.0b013e318292b7b9)

21. Maratt JD, Esposito Cl, McLawhorn AS, Jerabek SA, Padgett DE \& Mayman DJ. Pelvic tilt in patients undergoing total hip arthroplasty: when does it matter? Journal of Arthroplasty 201530 387-391. (https://doi.org/10.1016/j.arth.2014.10.014)

22. Rousseau MA, Lazennec JY, Boyer P, Mora N, Gorin M \& Catonné Y. Optimization of total hip arthroplasty implantation: is the anterior pelvic plane concept valid? Journal of Arthroplasty 200924 22-26. (https://doi.org/10.1016/j.arth.2007.12.015)

23. Loppini M, Longo UG, Ragucci P, Trenti N, Balzarini L \& Grappiolo G. Analysis of the pelvic functional orientation in the sagittal plane: a radiographic study with EOS 2D/3D technology. Journal of Arthroplasty 201732 1027-1032. (https://doi. org/10.1016/j.arth.2016.09.015)

24. Buckland AJ, Miyamoto R, Patel RD, Slover J \& Razi AE. Differentiating hip pathology From lumbar spine pathology: key points of evaluation and management. Journal of the American Academy of Orthopaedic Surgeons 201725 e23-e34. (https://doi. org/10.5435/JAAOS-D-15-00740)

25. Duval-Beaupère $\mathbf{G}$, Schmidt $\mathbf{C}$ \& Cosson $\mathbf{P}$. A Barycentremetric study of the sagittal shape of spine and pelvis: the conditions required for an economic standing position. Annals of Biomedical Engineering 199220 451-462. (https://doi.org/10.1007/ BF02368136)

26. Chuang CY, Liaw MY, Wang LY, Huang YC, Pong YP, Chen CW, Wu RW \& Lau YC. Spino-pelvic alignment, balance, and functional disability in patients with lowgrade degenerative lumbar spondylolisthesis. Journal of Rehabilitation Medicine $2018 \mathbf{5 0}$ 898-907. (https://doi.org/10.2340/16501977-2489)

27. Staibano P, Winemaker M, Petruccelli D \& de Beer J. Total joint arthroplasty and preoperative low back pain. Journal of Arthroplasty 201429 867-871. (https://doi. org/10.1016/j.arth.2013.10.001) 
28. Barbier 0, Skalli W, Mainard L, Mainard D \& Computer Assisted Orthopedic Surgery-France (CAOS-France). The reliability of the anterior pelvic plane for computer navigated acetabular component placement during total hip arthroplasty: prospective study with the EOS imaging system. Orthopaedics and Traumatology, Surgery and Research 2014 100 (Supplement) S287-S291. (https://doi.org/10.1016/j.otsr.2014.07.003)

29. Dubousset J, Charpak G, Skalli W, Kalifa G \& Lazennec JY. EOS stereoradiography system: whole-body simultaneous anteroposterior and lateral radiographs with very low radiation dose. Revue de Chirurgie Orthopédique et Reparatrice de l'Appareil Moteur 200793 (Supplement) 141-143. (https://doi.org/10.1016/s0035-1040(07)92729-4)

30. Kalifa G, Charpak Y, Maccia C, Fery-Lemonnier E, Bloch J, Boussard JM, Attal M, Dubousset J \& Adamsbaum C. Evaluation of a new low-dose digital x-ray device: first dosimetric and clinical results in children. Pediatric Radiology $1998 \mathbf{2 8}$ 557-561. (https://doi.org/10.1007/s002470050413)

31. Lazennec JY, Rangel A, Baudoin A, Skalli W, Catonne Y \& Rousseau MA. The EOS imaging system for understanding a patellofemoral disorder following THR. Orthopaedics and Traumatology, Surgery and Research 201197 98-101. (https://doi. org/10.1016/j.otsr.2010.07.010)

32. Lazennec JY, Boyer P, Gorin M, Catonné Y \& Rousseau MA. Acetabular anteversion with CT in supine, simulated standing, and sitting positions in a THA patient population. Clinical Orthopaedics and Related Research 2011469 1103-1109. (https://doi. org/10.1007/s11999-010-1732-7)

33. Lazennec JY, Charlot N, Gorin M, Roger B, Arafati N, Bissery A \& Saillant $\mathbf{G}$. Hip-spine relationship: a radio-anatomical study for optimization in acetabular cup positioning. Surgical and Radiologic Anatomy 200426 136-144. (https://doi. org/10.1007/s00276-003-0195-x)

34. Lazennec JY, Brusson A \& Rousseau M-A. Hip-Spine Relations: An Innovative Paradigm in THR Surgery. Intech0pen, 2012. (https://doi.org/10.5772/27544)

35. Vigdorchik JM, Sharma AK, Jerabek SA, Mayman DJ \& Sculco PK. Templating for total hip arthroplasty in the modern age. Journal of the American Academy of Orthopaedic Surgeons 202129 e208-e216. (https://doi.org/10.5435/JAAOS-D-20-00693)

36. Miyoshi H, Mikami H, $\mathbf{O b a}$ K \& Amari R. Anteversion of the acetabular component aligned with the transverse acetabular ligament in total hip arthroplasty. Journal of Arthroplasty 201227 916-922. (https://doi.org/10.1016/j.arth.2011.10.017)

37. Meftah M, Yadav A, Wong AC, Ranawat AS \& Ranawat CS. A novel method for accurate and reproducible functional cup positioning in total hip arthroplasty. Journal of Arthroplasty 201328 1200-1205. (https://doi.org/10.1016/j.arth.2012.09.018)

38. Archbold HAP, Mockford B, Molloy J, McConway J, Ogonda L \& Beverland $\mathbf{D}$. The transverse acetabular ligament: an aid to orientation of the acetabular component during primary total hip replacement: a preliminary study of 1000 cases investigating postoperative stability. Journal of Bone and Joint Surgery $2006 \mathbf{8 8} 883-886$. (https://doi.org/10.1302/0301-620X.88B7.17577)

39. Meermans G, Van Doorn WJ, Koenraadt K \& Kats J. The use of the transverse acetabular ligament for determining the orientation of the components in total hip replacement: a randomised controlled trial. Bone and Joint Journal 2014 96-B 312-318. (https://doi.org/10.1302/0301-620X.96B3.32989)

40. Gkiatas I, Karasavvidis T, Sharma AK, Xiang W, Malahias M-A, Chalmers BP, Jerabek SA \& Sculco PK. Use of elevated liners in primary total hip arthroplasty: a systematic review of the literature. European Journal of Orthopaedic Surgery and Traumatology 2021 In press. (https://doi.org/10.1007/s00590-021-03023-y)
41. Weber M, Meyer M, von Eisenhart-Rothe R \& Renkawitz $T$. The superiority of navigation and robotics in hip arthroplasty: fact or myth? Der Orthopade $202150270-277$. (https://doi.org/10.1007/s00132-021-04079-8)

42. Colombi A, Schena D \& Castelli CC. Total hip arthroplasty planning. EFORT Open Reviews 20194 626-632. (https://doi.org/10.1302/2058-5241.4.180075)

43. Dorr LD, Malik A, Dastane M \& Wan Z. Combined anteversion technique for total hip arthroplasty. Clinical Orthopaedics and Related Research 2009467 119-127. (https:// doi.org/10.1007/s11999-008-0598-4)

44. Maruyama M, Feinberg JR, Capello WN \& D’Antonio JA. The Frank Stinchfield Award: morphologic features of the acetabulum and femur: anteversion angle and implant positioning. Clinical Orthopaedics and Related Research 2001393 52-65.

45. Dorr LD, Faugere MC, Mackel AM, Gruen TA, Bognar B \& Malluche HH Structural and cellular assessment of bone quality of proximal femur. Bone 199314 231-242. (https://doi.org/10.1016/8756-3282(93)90146-2)

46. Loppini M, Longo UG, Caldarella E, Rocca AD, Denaro V \& Grappiolo G Femur first surgical technique: a smart non-computer-based procedure to achieve the combined anteversion in primary total hip arthroplasty. BMC Musculoskeletal Disorders 2017 18331 . (https://doi.org/10.1186/s12891-017-1688-9)

47. Pierrepont J, Yang L, Arulampalam J, Stambouzou C, Miles B \& Li Q The effect of seated pelvic tilt on posterior edge-loading in total hip arthroplasty: a finite element investigation. Proceedings of the Institution of Mechanical Engineers: Part H, Journal of Engineering in Medicine 2018232 241-248. (https://doi. org/10.1177/0954411917752028)

48. Traina F, De Fine $\mathbf{M}$, Bordini $\mathbf{B}$ \& Toni $\mathbf{A}$. Risk factors for ceramic liner fracture after total hip arthroplasty. Hip International 201222 607-614. (https://doi.org/10.5301/ HIP.2012.10339)

49. Dalla Pria P, Zagra L, Esopi P \& Masoni D. Breakage and noises in ceramic on ceramic couplings. European Orthopaedics and Traumatology 20101 53-59. (https://doi. org/10.1007/s12570-010-0015-9)

50. Zagra L \& Gallazzi E. Bearing surfaces in primary total hip arthroplasty. EFORT Open Reviews 2018 3 217-224. (https://doi.org/10.1302/2058-5241.3.180300)

51. De Martino I, D’Apolito R, Soranoglou VG, Poultsides LA, Sculco PK \& Sculco TP. Dislocation following total hip arthroplasty using dual mobility acetabular components: a systematic review. Bone and Joint Journal 2017 99-B 18-24. (https://doi. org/10.1302/0301-620X.99B1.BJJ-2016-0398.R1)

52. D'Apolito R, Bandettini G, Jacquot FMP \& Zagra L. Modular dual-mobility cups using ceramic liners: an original solution for selected indications? Hip International 2020 30 59-65. (https://doi.org/10.1177/1120700020964976)

53. Reina N, Pareek A, Krych AJ, Pagnano MW, Berry DJ \& Abdel MP. Dualmobility constructs in primary and revision total hip arthroplasty: a systematic review of comparative studies. Journal of Arthroplasty 201934 594-603. (https://doi.org/10.1016/j. arth.2018.11.020)

54. McCarthy TF, Alipit V, Nevelos J, Elmallah RK \& Mont MA. Acetabular cup anteversion and inclination in hip range of motion to impingement. Journal of Arthroplasty 201631 264-268. (https://doi.org/10.1016/j.arth.2016.01.067)

55. Ochi H, Baba T, Homma Y, Matsumoto M, Nojiri H \& Kaneko K. Importance of the spinopelvic factors on the pelvic inclination from standing to sitting before total hip arthroplasty. European Spine Journal 201625 3699-3706. (https://doi.org/10.1007/ s00586-015-4217-2) 
56. DelSole EM, Vigdorchik JM, Schwarzkopf R, Errico TJ \& Buckland AJ. Total hip arthroplasty in the spinal deformity population: does degree of sagittal deformity affect rates of safe zone placement, instability, or revision? Journal of Arthroplasty $2017 \mathbf{3 2}$ 1910-1917. (https://doi.org/10.1016/j.arth.2016.12.039)

57. Buckland AJ, Puvanesarajah V, Vigdorchik J, Schwarzkopf R, Jain A, Klineberg EO, Hart RA, Callaghan JJ \& Hassanzadeh H. Dislocation of a primary total hip arthroplasty is more common in patients with a lumbar spinal fusion. Bone and Joint Journal 2017 99-B 585-591. (https://doi.org/10.1302/0301-620X.99B5.BJJ-2016-0657.R1)

58. Sing DC, Barry JJ, Aguilar TU, Theologis AA, Patterson JT, Tay BK, Vail TP \& Hansen EN. Prior lumbar spinal arthrodesis increases risk of prosthetic-related complication in total hip arthroplasty. Journal of Arthroplasty $2016 \mathbf{3 1}$ (Supplement) 227. e1-232.e1. (https://doi.org/10.1016/j.arth.2016.02.069)

59. Diebo BG, Beyer GA, Grieco PW, Liu S, Day LM, Abraham R, Naziri Q, Passias PG, Maheshwari AV \& Paulino CB. Complications in patients undergoing spinal fusion after THA. Clinical Orthopaedics and Related Research 2018476 412-417. (https://doi.org/10.1007/s11999.0000000000000009)

60. Di Martino A, Bordini B, Ancarani C, Viceconti M \& Faldini C. Does total hip arthroplasty have a higher risk of failure in patients who undergo lumbar spinal fusion? Bone and Joint Journal 2021 103-B 486-491. (https://doi.org/10.1302/0301-620X.103B3. BJJ-2020-1209.R1)

61. Malkani AL, Himschoot KJ, Ong KL, Lau EC, Baykal D, Dimar JR, Glassman SD \& Berry DJ. Does timing of primary total hip arthroplasty prior to or after lumbar spine fusion have an effect on dislocation and revision rates? Journal of Arthroplasty 201934 907-911. (https://doi.org/10.1016/j.arth.2019.01.009)

62. Hinman AD, Inacio MCS, Prentice HA, Kuo CC, Khatod M, Guppy KH \& Paxton EW. Lumbar spine fusion patients see similar improvements in physical activity level to non-spine fusion patients following total hip arthroplasty. Journal of Arthroplasty 202035 451-456. (https://doi.org/10.1016/j.arth.2019.08.053) 